\title{
THE EXACT SEQUENCE OF LOW DEGREE AND NORMAL ALGEBRAS
}

\author{
BY LINDSAY N. CHILDS
}

\author{
Communicated by I. N. Herstein, February 18, 1970
}

The exact sequence of low degree associated to a first quadrant bicomplex (five terms long in [4, I.4.5.1] seven terms long in [2, Lemma 7.5]) has been used in a number of situations, for example, in obtaining a cohomological description of the Brauer group of a commutative ring $R[2]$. In this note we observe that the sequence may be extended to an infinitely long exact sequence. The terms arising from the homology of the total complex are not $F^{n-1} H^{n}$ (tot), the $(n-1)$ th filtration group of $H^{n}$, for $n>2$, but map onto it.

As an application we embed the seven term Galois cohomology sequence of $[1,5.5]$ into an infinite sequence, and sketch a map from normal Azumaya algebras into the eighth term which extends the Teichmüller cocycle map of [3].

1. Suppose given a bicomplex $\left\{C_{p, q}\right\}$ of abelian groups [5, p. 340] such that $C_{p, q}=0$ if $p<0$ or $q<0$. The differentials $d^{\prime}: C_{p, q} \rightarrow C_{p+1, q}$ and $d^{\prime \prime}: C_{p, q} \rightarrow C_{p, q+1}$ of the bicomplex, defined for all integers $p, q$, satisfy the conditions $d^{\prime} d^{\prime}=0, d^{\prime \prime} d^{\prime \prime}=0, d^{\prime} d^{\prime \prime}+d^{\prime \prime} d^{\prime}=0$. (Notation: $\operatorname{cl}\left(\right.$ ) will denote "cohomology class of.") Then $Z_{p, q}^{2}$ is the set of classes $\operatorname{cl}(u)$ in $\operatorname{ker}\left(d^{\prime \prime}\right) / \operatorname{im}\left(d^{\prime \prime}\right)$ such that $u$ is in $C_{p, q}, d^{\prime \prime}(u)=0$, and $d^{\prime}(u)$ $=d^{\prime \prime}(v)$ for some $v$ in $C_{p+1, q-1} ; B_{p, q}^{2}$ is the set of classes $\mathrm{cl}(u)$ such that $u$ in $C_{p, q}$ is of the form $u=d^{\prime}(v)+d^{\prime \prime}(w)$ with $d^{\prime \prime}(v)=0$; and $E_{p, q}^{2}$ $=Z_{p, q}^{2} / B_{p, q}^{2}$.

The $n$th group $C_{n}$ (tot) of the total complex $(n \geqq 0)$ is the group $C_{0, n} \oplus C_{1, n-1} \oplus \cdots \oplus C_{n-1,1} \oplus C_{n, 0}$. Set $D=d^{\prime \prime}+d^{\prime}$, the differential of the total complex $\left\{C_{n}\right.$ (tot) $\}$. Denote by $Z^{n}$ the elements of $C_{n}$ (tot) of the form $x=(0, \cdots, 0, u, v)$ with $D x=0$. Denote by $\dot{B}^{n}$ the elements of $Z^{n}$ of the form $x=D y$, where $y=\left(0, \cdots, 0, z_{1}, z_{0}\right)$ $\in C_{n-1}$ (tot), and $B^{n}$ the elements of $Z^{n}$ of the form $x=D y$ where $y=\left(z_{n}, z_{n-1}, \cdots, z_{2}, z_{1}, z_{0}\right) \in C_{n-1}($ tot $)$. Then the filtered group $F^{n-1} H^{n}$ of the total complex associated to the bicomplex is $Z^{n} / B^{n}$. We denote by $\dot{H}^{n}$ the group $Z^{n} / \dot{B}^{n}$. Note that there is clearly an epimorphism from $\dot{H}^{n}$ onto $F^{n-1} H^{n}$ for all $n$, and for $n=2$ or 1 it is the

AMS subject classifications. Primary 1616, 1840; Secondary 1672, 1320, 1810, 1205.

Key words and phrases. Azumaya algebras, bicomplex of abelian groups, Galois cohomology, normal central simple algebras, exact sequence of low degree. 
identity map.

THEOREM 1. The long exact sequence of low degree is

$$
\begin{aligned}
0 & \rightarrow E_{1,0}^{2} \rightarrow \dot{H}^{1} \rightarrow E_{0,1}^{2} \rightarrow E_{2,0}^{2} \rightarrow \dot{H}^{2} \rightarrow E_{1,1}^{2} \rightarrow \cdots \\
\cdots & \rightarrow E_{n, 0}^{2} \rightarrow \dot{H}^{n} \rightarrow E_{n-1,1}^{2} \rightarrow E_{n+1,0}^{2} \rightarrow \dot{H}^{n+1} \rightarrow \cdots .
\end{aligned}
$$

The first seven terms of this sequence are identical with the sequences of [2, Lemma 7.5].

The maps are as follows:

From $E_{n, 0}^{2}$ to $\dot{H}^{n}$ the map is obtained by sending $\operatorname{cl}(\operatorname{cl}(v))$ in $E_{n, 0}^{2}$ to the class of the element $(0, \cdots, 0, v)$ of $Z^{n}$. From $\dot{H}^{n}$ to $E_{n-1,1}^{2}$ the map is obtained by sending the class of an element $(0, \cdots, 0, u, v)$ of $Z^{n}$ to $\operatorname{cl}(\operatorname{cl}(u))$ in $E_{n-1,1}^{2}$. From $E_{n-1,1}^{2}$ to $E_{n+1,0}^{2}$ the map is obtained by sending $\operatorname{cl}(\operatorname{cl}(u))$ in the former to $\operatorname{cl}(\operatorname{cl}(w))$ in the latter, where $d^{\prime}(u)$ $=d^{\prime \prime}(z)$ for some $z$ in $C_{n, 0}$ and $w=d^{\prime}(z)$.

The proof of exactness is a routine computation.

Alternatively, one can obtain the sequence by forming the bicomplex $\left\{\dot{C}_{p, q}\right\}: \dot{C}_{p, q}=C_{p, q}$ for $q \leqq 1, \dot{C}_{p, q}=0$ for $q \geqq 3$, and $\dot{C}_{p, 2}$ $=d^{\prime \prime}\left(C_{p, 1}\right)$. Then one has a long exact sequence by [6, Corollary 2.3], whose $E^{2}$ terms are the same as those in Theorem 1 and it is not difficult to show that the $H^{n}$ (tot) of the bicomplex $\dot{C}_{p, q}$ occurring in this latter sequence is the same as the $\dot{H}^{n}$ of Theorem 1 . We omit details in either case.

2. We now sketch a generalization of the Teichmuller cocycle map for normal central simple algebras described by Eilenberg and MacLane [3]. For Amitsur cohomology and other unexplained notation see [2].

Let $S$ be a commutative ring with unit, and a Galois extension of $R$ with group $Q$ [1]; let $A$ be a Azumaya (=central separable) $S$ algebra. Suppose $A$ is $Q$-normal, i.e. there is a 1-1 set map $w$ from $Q$ to $\operatorname{Aut}_{R}(A)$ whose image restricts to $Q$ on $S$.

Let $K$ be a commutative $R$-algebra which is a faithfully flat $R$ module. Let $U_{S}$ be the functor from $R$-algebras to abelian groups defined by $U_{S}(K)=$ the multiplicative group of units of $S \otimes_{R} K$. Let $C_{p, q}=\left(Q^{p}, U_{S}\left(K^{q+1}\right)\right)$, with $p, q \geqq 0$, where (_, - _ ) denotes set maps, $Q^{p}$ denotes direct product $p$ times, $Q^{0}=\{1\}, K^{q+1}$ denotes tensor product over $R q+1$ times. Let $d_{Q}$ denote the group cohomology coboundary (in the $p$-direction), and $d_{K}^{\prime}$ the Amitsur cohomology coboundary (in the $q$-direction). Then $d_{K}^{\prime} d_{Q}=d_{Q} d_{K}^{\prime}$, so replacing $d_{K}^{\prime}$ by $s^{p} \circ d^{\prime}=d_{K}$ on $C_{p, q}$, where $s^{p}$ denotes the inverse map in $U_{S}$ applied $p$ times, we obtain a bicomplex. From it we obtain a long ex- 
act sequence of low degree. Part of it reads

$$
\begin{aligned}
\cdots & \rightarrow H^{1}\left(Q, H^{1}\left(K / R, U_{S}\right)\right) \rightarrow H^{3}\left(Q, H^{0}\left(K / R, U_{S}\right)\right) \\
& \rightarrow \dot{H}^{3}(\text { tot }) \rightarrow H^{2}\left(Q, H^{1}\left(K / R, U_{S}\right)\right) \rightarrow \cdots
\end{aligned}
$$

(We note that $H^{1}\left(K / R, U_{S}\right)$, Amitsur cohomology, is isomorphic to the kernel of the map from $\operatorname{Pic}(S)$ to $\operatorname{Pic}\left(S \otimes_{R} K\right)$ by descent, and $H^{0}\left(K / R, U_{S}\right)=U_{S}(R)=U(S)$, so that taking limits over faithfully flat $K$ (as in $[2, \S 6]$ ) yields, except for identifying inj $\lim \dot{H}^{2}$ (tot) with $B(S / R)$, an extension of the Chase-Harrison-RosenbergAuslander-Brumer seven term exact sequence of Galois cohomology $[1,5.5]$. Note that inj $\lim \dot{H}^{3}$ (tot) is the first term missing from $[1,5.5]$.)

Cocycles in $Z^{3}$ corresponding to elements of $\dot{H}^{3}$ (tot) are pairs $(f, g), f$ in $\left(Q^{2}, U_{S}\left(K^{2}\right)\right), g$ in $\left(Q^{3}, U_{S}(K)\right)$ where $d_{K}(f)=1, d_{K}^{\prime}(g)=d_{Q}(f)$ and $d_{Q}(g)=1$.

THEOREM 2. There is a well-defined map from the set of isomorphism classes of $Q$-normal Azumaya $S$-algebras into inj $\lim _{K} \dot{H}^{3}$ (tot). When $S$ is semilocal $\dot{H}^{3}($ tot $) \cong \dot{H}^{3}(G, U(S))$ and the composition is the Teichmuller cocycle map of [3].

We shall restrict ourselves here to sketching the definition of the map, and leave checking of the well-definedness (which is not difficult) and elaboration of the theory for future appearance elsewhere.

The map is defined as follows. Let $w: Q \rightarrow \mathrm{Aut}_{R}(A)$ be an extension of $Q$ to $A$. Then $d w(\lambda, \mu)=w(\lambda) w(\mu) w(\lambda, \mu)^{-1}$ fixes $S$ so is an element of $\operatorname{Aut}_{S}(A)$. We have a map $J: \operatorname{Aut}_{S}(A) \rightarrow \operatorname{Pic}(S)$ defined by: $f$ goes to $J_{f}=\{a$ in $A \mid f(x) a=a x$ for all $x$ in $A\}$. So $d w$ yields a map $J_{d w}$ from $Q^{2}$ to $\operatorname{Pic}(S)$. (See [7].)

Let $K$ be a faithfully flat $R$-algebra such that $S \otimes_{R} K$ splits all the images of $J_{d w}$. Viewing $d w$ as mapping $Q^{2}$ into Aut $\mathrm{t}_{S \otimes K}(A \otimes K)$, since $J_{d w(\lambda, \mu)} \otimes K$ is a free $S \otimes K$ module with basis element $u(\lambda, \mu)$ in $A \otimes K$, $d w(\lambda, \mu)$ is conjugation by $u(\lambda, \mu)$ in $A \otimes K$ [7].

An element $f$ of $\left(Q^{2}, U\left(S \otimes K^{2}\right)\right)$ corresponding to $A$ is the Amitsur cocycle corresponding to the modules $J_{d w(\lambda, \mu)}$, namely $d_{K}^{\prime}(u)$ where

$$
d_{K}^{\prime}(u(\lambda, \mu))=\epsilon_{1}(u(\lambda, \mu))^{-1} \epsilon_{0}(u(\lambda, \mu)) \text {. }
$$

On the other hand, an element $g$ of $\left(Q^{3}, U_{S}(K)\right)$ corresponding to $A$ is the noncommutative coboundary $d_{Q}(u)$, defined exactly as the Teichmüller cocycle is defined in the classical case [3], viz:

$$
d_{Q}(u)(\lambda, \mu, \nu)=u(\mu, \nu)^{w(\lambda)} u(\lambda, \mu \nu) u^{-1}(\lambda \mu, \nu) u^{-1}(\lambda, \mu) .
$$


It is not difficult to show that $d_{K}^{\prime}\left(d_{Q}(u)\right)=d_{Q}\left(d_{K}^{\prime}(u)\right)$, so that $\left(d_{K}(u), d_{Q}(u)\right)$ is an element of $Z^{3}$, whose class in $\dot{H}^{3}$ is the image of $A$ under the map of Theorem 2.

\section{REFERENCES}

1. S. U. Chase, D. K. Harrison and A. Rosenberg, Galois theory and Galois cohomology of commutative rings, Mem. Amer. Math. Soc. No. 52 (1965), 15-33. MR 33 \#4118.

2. S. U. Chase and A. Rosenberg, Amitsur cohomology and the Brauer group, Mem. Amer. Math. Soc. No. 52 (1965), 34-79. MR 33 \#4119.

3. S. Eilenberg and S. Mac Lane, Cohomology and Galois theory. I: Normality of algebras and Teichmuiller's cocycle, Trans. Amer. Math. Soc. 64 (1948), 1-20. MR 10, 5.

4. R. Godement, Theorie des faisceaux, Hermann, Paris, 1964.

5. S. Mac Lane, Homology, Die Grundlehren der math. Wissenschaften, Band 114, Academic Press, New York, and Springer-Verlag, Berlin, 1963. MR 28 \#122.

6. B. Mitchell, Spectral sequences for the layman, Amer. Math. Monthly 76 (1969), 599-605. MR 39 \#4848.

7. A. Rosenberg and D. Zelinsky, Automorphisms of separable algebras, Pacific J. Math. 11 (1961), 1109-1117. MR $26 \# 6215$.

State University of New York, Albany, New York 12203 